\title{
Multicellularity of Delicate Topological Insulators
}

\author{
Aleksandra Nelson $\odot,{ }^{1, *}$ Titus Neupert $\odot,{ }^{1}$ Tomáš Bzdušek $\odot,{ }^{2,1}$ and A. Alexandradinata $\odot^{3, \dagger}$ \\ ${ }^{1}$ Department of Physics, University of Zurich, Winterthurerstrasse 190, 8057 Zurich, Switzerland \\ ${ }^{2}$ Condensed Matter Theory Group, Paul Scherrer Institute, 5232 Villigen PSI, Switzerland \\ ${ }^{3}$ Department of Physics, University of Illinois at Urbana-Champaign, Urbana, Illinois 61801-2918, USA
}

(Received 24 September 2020; accepted 21 April 2021; published 26 May 2021)

\begin{abstract}
Being Wannierizable is not the end of the story for topological insulators. We introduce a family of topological insulators that would be considered trivial in the paradigm set by the tenfold way, topological quantum chemistry, and the method of symmetry-based indicators. Despite having a symmetric, exponentially localized Wannier representation, each Wannier function cannot be completely localized to a single primitive unit cell in the bulk. Such multicellular topology is shown to be neither stable nor fragile, but delicate; i.e., the topology can be nullified by adding trivial bands to either valence or conduction band.
\end{abstract}

DOI: 10.1103/PhysRevLett.126.216404

Introduction.-Two themes have indelibly shaped the paradigm of topological insulators (TIs) and couched how topological properties are discussed, modeled, and measured. The first is the notion of stability of TIs, and the second involves the various obstructions to forming a realspace Wannier-function (WF) representation of the valence band [1-8]. This Letter describes an extension and finegraining of both themes and introduces a novel family of TIs that would be considered unstable and unobstructed according to the presently held paradigm.

The strongest form of stability is the notion of stable equivalence introduced by $K$ theory [8-12], where the bulk or surface topological invariant of a valence subspace is immune to addition of trivial bands. The intermediate notion of fragility means that the topological property can be nullified by adding trivial bands to the valence subspace, but not to the conduction subspace [13-19] [Fig. 1(a)]. A distinct notion that we introduce here is delicate topology, where the topological property can be nullified by adding trivial bands to either valence or conduction subspace [Fig. 1(b)]. For symmetry-protected delicate topology, nullification occurs only by adding trivial bands of certain symmetry representations.

Many authors have proposed a useful definition of a trivial band to be its possession of an exponentially localized WF representation respecting the crystallographic spacetime symmetries [11,12,19-23]. By this definition, all stably equivalent and fragile TIs present an obstruction to a WF representation. It has been further argued through equivariant vector bundle theory that such Wannier obstructions represent a robust property of a valence subspace summed with an arbitrary conduction subspace [19], and therefore such obstruction cannot exist for delicate topological insulators. Here, we introduce a distinct class of obstructions that prevents WFs from being completely localized to a single, primitive unit cell-we call this multicellular topology [Figs. 2(a)-2(c)]. Conversely, we adopt a distinct notion of triviality, namely that symmetryrespecting WFs exist and can be confined to a single cell by a continuous, adiabatic deformation of the Hamiltonianunicellularity.

The notions of delicate and multicellular topology are distinct and a priori need not come together in any specific realization of a TI. This Letter aims to open the debate by presenting a concrete family of tight-binding models that simultaneously manifests both types of topology and sets the stage for future realizations and discoveries.

Returning Thouless pump.-We begin by introducing a class of tight-binding models in three spatial dimensions, which exhibit both symmetry-protected delicacy and multicellularity. The relevant symmetry is an $n$-fold rotation symmetry $C_{n}$ about the $z$ axis. The tight-binding Hilbert space is given by an orthonormal set of WFs $\left\{\varphi_{j, \boldsymbol{R}}\right\}_{\boldsymbol{R} \in \mathrm{BL}, j=1, \ldots, \mathcal{C}+\mathcal{V}}$ over the Bravais lattice (BL), which satisfy the uniaxial condition, i.e., that all independent WFs

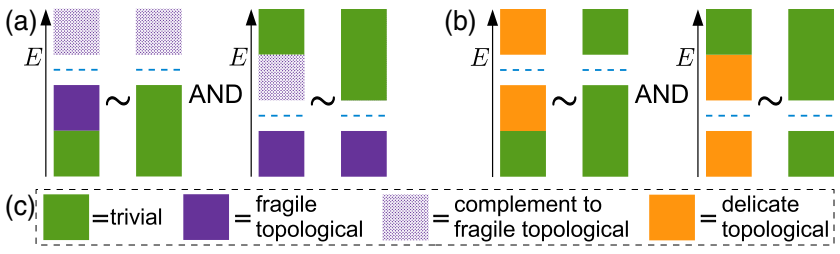

FIG. 1. Topological stability of (a) fragile vs (b) delicate topology. The blocks [colored according to legend in (c)] below (above) the Fermi energy (dashed blue lines) are valence (conduction) bands, with addition indicated by stacking and topological equivalence by " $\sim$." In panel (a) the complement to fragile topological bands could be fragile topological, obstructed atomic limit, or fully trivial. 

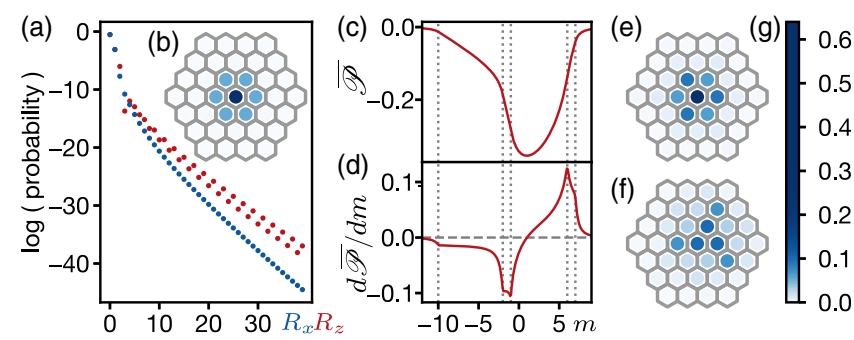

FIG. 2. Characterization of (a)-(d) bulk and (e),(f) surface Wannier functions for the delicate topological insulator modeled by Eq. (1) with $m=-6, \chi=-1$. (a) illustrates the exponential decay parallel (orthogonal) to the rotation axis, as indicated by $R_{z}$ $\left(R_{x}\right)$. (b),(e),(f) Rotation-symmetric probability distributionprojected onto the rotation-invariant plane, utilizing the color bar in (g). (c),(d) The total polarization in $z$ direction is nonanalytic at topological-phase transitions indicated by dashed lines.

within a representative, primitive unit cell are centered on the same rotational axis and individually form onedimensional representations of $C_{n}$. (This simplifying assumption holds only for the subclass of multicellular TIs studied here.) This allows one to decompose the Hilbert space as $\mathscr{H}[\varphi]=\oplus_{\ell=0}^{n-1} \mathscr{H}_{\ell}[\varphi]$, where the summands are distinguished by the $n$ possible angular momenta $\ell$, with corresponding rotation eigenvalues $e^{i 2 \pi \ell / n}$.

We further assume the valence (respectively, conduction) bands can be spanned by exponentially localized WFs $\left\{W_{j, \boldsymbol{R}}^{v}\right\}_{\boldsymbol{R} \in \mathrm{BL}, j=1, \ldots, \mathcal{V}} \quad$ (respectively, $\left\{W_{j, \boldsymbol{R}}^{c}\right\}_{\boldsymbol{R} \in \mathrm{BL}, j=1, \ldots, \mathcal{C}}$ ). Though generally distinct from $\left\{\varphi_{j, R}\right\}$, we demand that $\left\{W_{j, \boldsymbol{R}}^{c / v}\right\}$ also satisfy the uniaxial condition and additionally satisfy the mutually disjoint condition - that any representation appearing in the valence subspace $\left(\mathscr{H}\left[W^{v}\right]=\oplus_{\ell_{v}}, \mathscr{H}_{\ell_{v}}\left[W^{v}\right]\right)$ cannot appear in the conduction subspace $\left(\mathscr{H}\left[W^{c}\right]=\oplus_{\ell_{c}} \mathscr{H}_{\ell_{c}}\left[W^{c}\right]\right.$, with $\ell_{v(c)}$ disjoint).

The uniaxial condition on exponentially localized WFs implies that both conduction and valence bands are band representations [24,25], making the system trivial from the viewpoints of topological quantum chemistry [20] and symmetry-based indicators [22]. A band representation also precludes a nontrivial first Chern class [19,23], making the model trivial in the tenfold way $[9,26,27]$. Nevertheless, we find that the mutually disjoint condition allows for a type of symmetry-protected multicellularity, where the WFs necessarily extend-beyond one unit cell-in the direction of the rotation axis.

The multicellularity manifests in the discrete spectrum of the projected position operator $P \hat{z} P[28,29]$, with $P$ projecting to the bulk valence band. Since $P \hat{z} P$ is invariant under translations perpendicular to the rotation axis, each eigenvalue of $P \hat{z} P$ forms a band over the two-dimensional (2D) reduced Brillouin zone, $r \mathrm{BZ} \ni \boldsymbol{k}_{\perp}=\left(k_{x}, k_{y}\right)$. Under translation along the rotation axis by a lattice period (set to one), $P \hat{z} P \rightarrow P(\hat{z}+1) P$, hence each eigenvalue belongs to an infinitely extended Wannier-Stark ladder [30], and the full spectrum comprises $\mathcal{V}$ such ladders, which are nondegenerate at generic $\boldsymbol{k}_{\perp}$ [7]. We pick one representative eigenvalue from each ladder and define their sum (modulo integer) to be the (charge) polarization $\mathscr{P}\left(\boldsymbol{k}_{\perp}\right)$, in accordance with the geometric theory of polarization $[31,32]$.

Since distinct rotational representations cannot mix at $C_{n}$-invariant points $\left(\boldsymbol{k}_{\perp}^{\prime} \equiv C_{n} \boldsymbol{k}_{\perp}^{\prime}\right)$, the polarization can be decomposed into a sum of polarizations in each angularmomentum sector: $\mathscr{P}\left(\boldsymbol{k}_{\perp}^{\prime}\right)=\sum_{\ell_{v}} \mathscr{P}_{\ell_{v}}\left(\boldsymbol{k}_{\perp}^{\prime}\right)$. This nonmixing, combined with the mutually disjoint condition, implies an identity between symmetry-decomposed Hilbert spaces $\left.\mathscr{H}_{\ell_{v}}\left[W^{v}\right]\right|_{\boldsymbol{k}_{\perp}^{\prime}}=\left.\mathscr{H}_{\ell_{v}}[\varphi]\right|_{\boldsymbol{k}_{\perp}^{\prime}}$ when restricted to any $C_{n^{-}}$ invariant wave vector. It follows that the polarization $\mathscr{P}_{\ell_{v}}\left(\boldsymbol{k}_{\perp}^{\prime}\right)$ equals, modulo integer, to the polarization of the basis WFs in the spin sector $\ell_{v}$; the latter quantity is $\boldsymbol{k}_{\perp}^{\prime}$ independent because any tight-binding basis function has support only on a single lattice site. Therefore, modulo integer, $\mathscr{P}_{\ell_{v}}\left(\boldsymbol{k}_{\perp}^{\prime}\right)$ is independent of $\boldsymbol{k}_{\perp}^{\prime}$, and hence also $\mathscr{P}\left(\boldsymbol{k}_{\perp}^{\prime}\right)$. If $\mathscr{P}\left(\boldsymbol{k}_{\perp}\right)$ is continuously defined over $r \mathrm{BZ}$ with multiple $C_{n}$-invariant points, the difference $\Delta \mathscr{P}_{\boldsymbol{k}_{\perp}^{\prime} \boldsymbol{k}_{\perp}^{\prime \prime}}:=$ $\mathscr{P}\left(\boldsymbol{k}_{\perp}^{\prime \prime}\right)-\mathscr{P}\left(\boldsymbol{k}_{\perp}^{\prime}\right)$ between any pair of these points is quantized to integers. $\Delta \mathscr{P}_{k_{\perp}^{\prime} k_{\perp}^{\prime \prime}}=\mu$ implies a Thouless pump [33] of $\mu$ electron charges over one half-period of the $r \mathrm{BZ}$ (connecting $\boldsymbol{k}_{\perp}^{\prime}$ and $\boldsymbol{k}_{\perp}^{\prime \prime}$ ); the triviality of the first Chern class ensures that this charge is reversed in the second half-period. Such a returning Thouless pump (RTP) guarantees that (i) the Hamiltonian cannot be adiabatically deformed to be $\boldsymbol{k}$ independent (having no hopping elements in real space), and (ii) at least one WF must extend over multiple unit cells in the direction of the rotation axis (see Supplemental Material [34]).

Minimal model.-To exemplify a nontrivial RTP, we consider a two-band, tight-binding model with sixfold $\left(C_{6}\right)$ rotational symmetry. On each site of a triangular lattice, we situate an $s$ and a $p_{+}=p_{x}+i p_{y}$ (spinless) orbital, which transform under $C_{6}$ with angular momenta $\ell_{v}=0$ and $\ell_{c}=1$, respectively. The Hamiltonian has the form

$$
\begin{aligned}
& H(\boldsymbol{k})=\left[z^{\dagger}(\boldsymbol{k}) \boldsymbol{\sigma} z(\boldsymbol{k})\right] \cdot \boldsymbol{\sigma}, \quad z(\boldsymbol{k})=\left(z_{1}, z_{2}\right)^{T}, \\
& z_{1}(\boldsymbol{k})=\sum_{a=1}^{6} e^{-i \pi a / 3} \exp \left[i \boldsymbol{t}(a) \cdot \boldsymbol{k}_{\perp}\right], \\
& z_{2}(\boldsymbol{k})=\sin k_{z}+i\left(\sum_{a=1}^{6} \exp \left[i \boldsymbol{t}(a) \cdot \boldsymbol{k}_{\perp}\right]+4 \cos k_{z}+m\right),
\end{aligned}
$$

where $\boldsymbol{t}(a)=[\cos (\pi a / 3), \sin (\pi a / 3)], m$ is a tuning parameter for topological-phase transitions, and $\sigma$ is the vector of Pauli matrices with $\left\langle\sigma_{z}\right\rangle=1$ (respectively, -1 ) corresponding to the $s$ (respectively, $p_{+}$) orbital. For generic $m \in \mathbb{R}$, an energy gap exists throughout the $\mathrm{BZ}$, and the conduction (respectively, valence) eigenvector is a periodic-in-BZ, 
analytic function $u^{c}(\boldsymbol{k})=z(\boldsymbol{k}) /\|z(\boldsymbol{k})\|$ [respectively, $\left.u^{v}(\boldsymbol{k})=i \sigma_{y} u^{c}(\boldsymbol{k})^{*}\right]$ satisfying the symmetry condition $U_{6} u^{v / c}(\boldsymbol{k})=\exp \left(i 2 \pi \ell_{v / c} / 6\right) u^{v / c}\left(R_{6} \boldsymbol{k}\right)$, with $U_{6}$ (respectively, $R_{6}$ ) the pseudospinor (defining) representation of $C_{6}$. Consequently, the mutually disjoint condition is satisfied with $u^{c}$ (respectively, $u^{v}$ ) being $p_{+}$-like (respectively, $s$-like) along all rotation-invariant lines, and $u^{c / v}$ Fourier transform to symmetric, exponentially decaying [42] WFs [Figs. 2(a) and 2(b)]. Applying our previous argument for the integer-quantization of $\Delta \mathscr{P}_{\boldsymbol{k}_{\perp}^{\prime} \boldsymbol{k}_{\perp}^{\prime \prime}}$, we find that the polarization at all $C_{2}$-invariant points $\left(\Gamma, M, M^{\prime}, M^{\prime \prime}\right)$ and $C_{3}$-invariant points $\left(\Gamma, K, K^{\prime}\right)$ in the $r \mathrm{BZ}$ [cf. Fig. 3(c)] are identical modulo integer. The sixfold symmetry implies there are two independent polarization differences $\Delta \mathscr{P}_{K \Gamma}$ and $\Delta \mathscr{P}_{M \Gamma}$.

For large $|m|$, the Hamiltonian reduces to a $\boldsymbol{k}$-independent diagonal form $H(\boldsymbol{k}) \approx-m^{2} \sigma_{z}$, implying that the $s$-type valence (and also the $p_{+}$-type conduction) band is unicellular. This is consistent with $\mathscr{P}\left(\boldsymbol{k}_{\perp}\right)$ being continuously deformable to a flat sheet for representative values $m=-11$ and $m=8$, as illustrated by the blue, respectively, brown line in Fig. 3(b). Increasing $m$ from -11 to -10 , the bulk gap closes at the Brillouin-zone center; the resultant effective-mass Hamiltonian has the form in Eq. (1) with $z_{1}=$ $3\left(k_{y}+i k_{x}\right)$ and $z_{2}=k_{z}+i(10+m)$, which identifies the quadratic band-touching point as a dipole source of Berry curvature [43] with dipole moment parallel to the rotationinvariant $\boldsymbol{k}$ line. This dipole intermediates [43] a valence-toconduction transfer of a $2 \pi$ quantum of the Berry-Zak phase $\left(\phi_{Z}\right)$ - defined for the parallel transport of Bloch functions along said $\boldsymbol{k}$ line. Since $\phi_{Z} / 2 \pi \equiv_{1} \mathscr{P}(\Gamma)$ according to the geometric theory of polarization [31,32], with $\equiv_{j}$ meaning "equal $(\bmod j)$," there is correspondingly a discontinuous unit decrease of $\Delta \mathscr{P}_{M \Gamma}$ and $\Delta \mathscr{P}_{K \Gamma}$ when the gap reopens for $\delta m:=m+10 \gtrsim 0$ [orange line in Fig. 3(b)]. This further manifests as a " $(\delta m)^{2} \operatorname{sgn}[\delta m]$ "-type nonanalyticity in the total polarization $\overline{\mathscr{P}}=\int d^{2} k_{\perp} \mathscr{P}\left(\boldsymbol{k}_{\perp}\right) / \operatorname{Area}(r \mathrm{BZ})$ [cf. Figs. 2(c) and 2(d)] [34]. Further gap closings (at $m=-2,-1,6,7)$ result in Berry dipoles at other highsymmetry wave vectors, with the resultant phase diagram and RTPs summarized in Figs. 3(a) and 3(b).
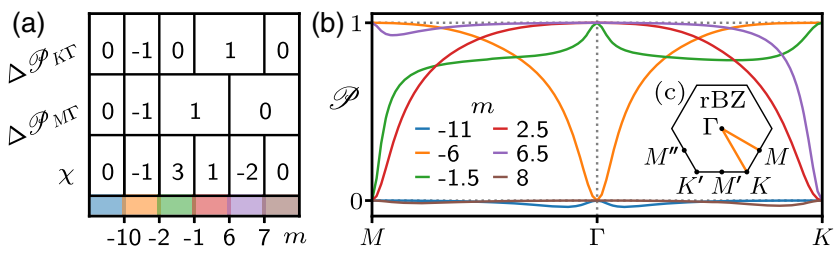

FIG. 3. (a) The polarization and Hopf invariants as a function of $m \in \mathbb{R}$ [cf. Eq. (1)]; colors distinguish distinct phases. (b) For each phase, the polarization along $M \Gamma K$ is plotted for a representative value of $m$. (c) Rotation-invariant points in reduced Brillouin zone.
Stability of RTP.—Equation (1) represents a minimal model of a RTP with the smallest dimension for the matrix $H(\boldsymbol{k})$. Models of arbitrarily large matrix dimensions can be constructed from our minimal model by adding unicellular bands to either conduction or valence subspace, assuming their symmetry representations maintain the mutually disjoint condition - this preserves the integer-valued quantization of $\Delta \mathscr{P}_{M \Gamma}$ and $\Delta \mathscr{P}_{K \Gamma}$, hence also the RTP. In contrast (as numerically verified in the Supplemental Material [34]), the quantization is lost upon addition of unicellular conduction bands that nullify the mutually disjoint condition, thus manifesting the RTP is a symmetry-protected delicate invariant.

Multicellularity with only translational symmetry.Which of our conclusions survive when rotational symmetry is relaxed? While the RTP generically destabilizes, we show that multicellularity persists - at least for the minimal model and any continuous deformation thereof that preserves the bulk energy gap and the bulk translational symmetry; any other symmetry can be relaxed. We appeal to a special feature of Pauli-matrix Hamiltonians with a spectral gap at each three-momentum $\boldsymbol{k}$; namely, that even with a trivial first Chern class, $H(\boldsymbol{k})$ has an integer-valued classification given by the Hopf invariant $\chi$ [44-48], which is equivalent to a Brillouin-zone integral of the Abelian Chern-Simons 3-form [48,49]

$$
\chi=-\frac{1}{4 \pi^{2}} \int_{\mathrm{BZ}} \boldsymbol{A} \cdot(\nabla \times \boldsymbol{A}) d^{3} k,
$$

with $\boldsymbol{A}(\boldsymbol{k})=\left\langle u \mid i \nabla_{\boldsymbol{k}} u\right\rangle$ the Berry connection of an energynondegenerate band [50]. Since $\chi$ is integer-quantized only for Pauli-matrix Hamiltonians, it is manifestly a delicate topological invariant distinct from RTP. That our minimal model for $m \in[-10,-2]$ has $\chi=-1$ is a consequence of a single Berry dipole intermediating a unit change in $\chi$ at $\Gamma$ at $m=-10$ [43].

That $\chi \neq 0$ implies multicellularity is now proven by contradiction. Assume that the valence-band WF is localizable to one unit cell, i.e., $W_{\boldsymbol{R}}^{v}=\delta_{\boldsymbol{R}, 0} \kappa_{v}$, with $\kappa_{v}$ a pseudospinor wave function that corresponds to a single point on the Bloch sphere $S^{2}$. The Fourier transform of $W_{\boldsymbol{R}}^{v}$ is then $\boldsymbol{k}$ independent, namely $u_{v}(\boldsymbol{k})=\kappa_{v}$. It is an eigenvector of a Hamiltonian that represents the trivial, constant map from the BZ to $S^{2}$, in contradiction with the assumed nontrivial Hopf invariant.

Hopf-RTP correspondence.-We have shown that both the Hopf invariant and RTP imply multicellularity. In fact, by a straightforward application of Whitehead's formulation of the Hopf invariant [51], we find [34] that the Hopf invariant and RTP are related to each other as [cf. Fig. 3(a)]

$$
\chi \equiv{ }_{6} 3 \Delta \mathscr{P}_{M \Gamma}-2 \Delta \mathscr{P}_{K \Gamma},
$$

for any $C_{6}$-symmetric, Pauli-matrix Hamiltonian having trivial Chern class and satisfying the uniaxial and mutually disjoint conditions with $\ell_{v}=0, \ell_{c}=1$. 
Bulk-boundary correspondence.-We have established the RTP and Hopf invariant as bulk delicate invariants leading to bulk multicellularity, but what does bulk multicellularity imply in the presence of a rotation-invariant surface termination? We answer with the following obstruction principle: there does not exist a symmetric, 2D tightbinding description (of a single surface facet) where all WFs are centered on the same rotational axes as the bulk WFs. Alternatively stated, on a half-infinite slab, the entire Hilbert space of states-filled and unfilled, bulk extended and surface localized - cannot be spanned by (uniaxially symmetric, exponentially localized) WFs whose positional centers coincide with the WFs obtained under periodic boundary conditions. (In contrast, for the "boundaryobstructed" topological phase studied in Ref. [52], a Wannier obstruction exists for the filled subspace but not for the entire Hilbert space.)

A stronger form of our principle is realized by the halfinfinite, Hopf-insulating slab (with or without rotational symmetry), namely that its Hilbert space does not even have an exponentially localized WF representation, because it is characterized [3] by a nonvanishing first Chern number-a stable, $K$-theoretic invariant [9]. This follows from the equality [43] of the bulk invariant $\chi$ and the faceted Chern number $\mathscr{C}_{f}$ - defined as the net Chern number of all surface-localized bands, independent of filling. The reason for this bulk-boundary correspondence is that bulk bands [characterized by a nontrivial ChernSimons 3-form of the Berry connection, cf. Eq. (2)] result in a surface anomalous Hall conductance (SAHC), according to the geometric theory of the magnetoelectric polarizability [53-58]; since the net SAHC of the entire Hilbert space must vanish, this necessitates the existence of surface bands that contribute a canceling SAHC [43]. Figure 4(a) illustrates the topologically nontrivial surface-localized band with Chern number $\mathscr{C}_{f}=-1$ for our minimal model ( $m=-6, \chi=-1)$; we emphasize that band(s) with the counterbalancing Chern number $\mathscr{C}_{f}^{\prime}=+1$ do not exist in the entire Hilbert space (of filled and unfilled states) on a half-infinite geometry.
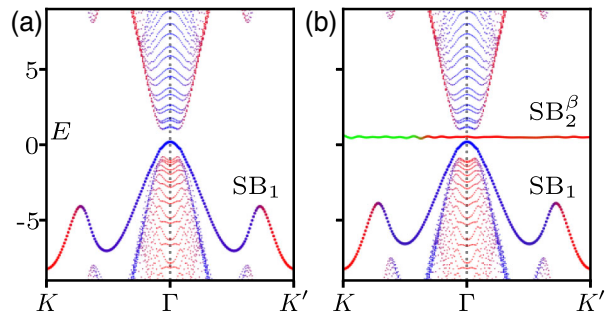

\begin{tabular}{l|c|c|c} 
(c) & $\ell_{\Gamma}$ & $\ell_{K}$ & $\ell_{K^{\prime}}$ \\
\hline \multirow{2}{*}{$\mathrm{VB}$} & 0 & 0 & 0 \\
& 2 & 2 & 2 \\
\hline $\mathrm{CB}$ & 1 & 1 & 1 \\
\hline $\mathrm{SB}_{1}$ & 1 & 0 & 0 \\
\hline $\mathrm{SB}_{2}^{\alpha}$ & 2 & 0 & 0 \\
$\mathrm{SB}_{2}^{\beta}$ & 0 & 2 & 0 \\
$\mathrm{SB}_{2}^{\gamma}$ & 0 & 0 & 2
\end{tabular}

FIG. 4. Spectrum of (a) Hopf-insulating and (b) RTP-insulating slab with one, respectively, two detached surface bands. Red, blue, green coloring represents contribution to the bands from basis orbitals with $\ell=0,1,2$. (c) For various bands discussed in the main text, $\ell_{\Gamma}, \ell_{K}, \ell_{K^{\prime}}$ denote the mod-three angular momenta at $C_{3}$-invariant wave vectors.
Next we demonstrate with an example that the Hilbert space of a half-infinite RTP insulator either has no 2D tightbinding description (owing to stable or fragile topology) or has a 2D tight-binding description with displaced Wannier centers. To model an insulator that is not a Hopf insulator and yet has a nontrivial RTP, we enlarge the Hilbert space of our minimal model $(m=-6)$ by adding a unicellular valence band whose representative WF has angular momentum $\ell=2$. To simplify the discussion, we restrict ourselves to the $P 3$ space group by including $C_{3}$-symmetric (and $C_{2}$-asymmetric) Hamiltonian matrix elements. By construction, the mutually disjoint condition is satisfied for representations of $C_{3}$, thus the polarization difference $\Delta \mathscr{P}_{K \Gamma}=-1$ remains quantized, but quantization no longer holds for $\Delta \mathscr{P}_{M \Gamma}$.

For the bulk valence (VB) and conduction (CB) bands, the symmetry representations at $C_{3}$-invariant wave vectors are presented in the first three rows of Fig. 4(c). The fourth row of Fig. 4(c) gives the symmetry representations of the nontrivial surface band $\mathrm{SB}_{1}$ [cf. Fig. 4(b)], which is topologically equivalent to the nontrivial surface band of the minimal model in Fig. 4(a). Observe that the representations of $\mathrm{SB}_{1}$ are identical to those of VBs except at $\Gamma$, where $\mathrm{SB}_{1}$ has the same representation as $\mathrm{CB}$. This may be rationalized by a thought experiment of imposing a surface termination on the bulk $s$-dominated WF; because of its nontrivial polarization [cf. Fig. 2(c)], such termination generates energetically unfavorable dangling bonds; to remove these bonds, the surface WF hybridizes with $p_{+}$type orbitals.

We are ready to diagnose the advertised obstruction: having Chern number $\mathscr{C}_{f}=-1, \mathrm{SB}_{1}$ has no exponentially localized WF representation. To attain such a representation, one must sum the surface band with another band over the $r \mathrm{BZ}$ having the opposite Chern number. Indeed, by modification of the surface Hamiltonian, one may always localize a second surface band $\mathrm{SB}_{2}$ by detaching it (i.e., "peeling it off") from either the VBs or CB. If detached from the $\mathrm{CB}, \mathrm{SB}_{2}$ combines bulk symmetry representations from the conduction subspace [third row in Fig. 4(c)]. For a $C_{3}$-symmetric band with Chern number $\mathscr{C}$, the product of $C_{3}$ eigenvalues at $\left\{\Gamma, K, K^{\prime}\right\}$ gives $e^{-i 2 \pi \mathscr{C} / 3}$ [59]. It follows that any detachment from $\mathrm{CB}$ necessarily has $\mathscr{C} \equiv_{3} 0$ and cannot nullify the unit Chern number of $\mathrm{SB}_{1}$. Instead, if we apply the same rule to detachments from VBs, we find three possible symmetry representations for $\mathrm{SB}_{2}$ with $\mathscr{C}=+1$, which we denote by $\mathrm{SB}_{2}^{\alpha, \beta, \gamma}$ in Fig. 4(c) and discuss in order.

Though a WF representation exists for the composite band $\mathrm{SB}_{1} \oplus \mathrm{SB}_{2}^{\alpha}$, these WFs cannot individually be $C_{3}$ symmetric on any of the $C_{3}$-invariant Wyckoff positions: $\{1 a, 1 b, 1 c\}$. Indeed, the symmetry representations of $\mathrm{SB}_{1} \oplus \mathrm{SB}_{2}^{\alpha}$ are incompatible with a band representation of space group $P 3$, which is deducible by comparison with symmetryrepresentation tables in the Bilbao crystallographic 
server [60]. The obstruction to $C_{3}$-symmetric WFs is fragile, in the sense that a trivial band $\mathrm{TB}$ exists (though not necessarily in the present Hilbert space), such that $\mathrm{SB}_{1} \oplus$ $\mathrm{SB}_{2}^{\alpha} \oplus \mathrm{TB}$ is not obstructed.

In contrast, by comparing the symmetry representations of $\mathrm{SB}_{1} \oplus \mathrm{SB}_{2}^{\beta}$ with the Bilbao tables, we deduce that $\mathrm{SB}_{1} \oplus \mathrm{SB}_{2}^{\beta}$ is a band representation with representative WFs of angular momentum $\ell=1$ and $\ell=0$, centered on the $1 c$ and $1 a$ Wyckoff positions, respectively [34] [Figs. 2(e) and 2(f)]; $\mathrm{SB}_{1} \oplus \mathrm{SB}_{2}^{\gamma}$ is likewise band representable with $\ell=1$ and $\ell=0$, centered on $1 b$ and $1 a$, respectively. Indeed, no matter how many bands are detached from $\mathrm{VBs}$ and added to $\mathrm{SB}_{1}$, the resultant, composite band cannot have a tight-binding description with all Wannier centers on the $1 a$ Wyckoff position of the bulk WFs. (In the language of topological quantum chemistry [20], the surface WFs realize an "obstructed atomic limit," while this is not true for the bulk WFs [34].) Assuming the contrary, the set of $C_{3}$ eigenvalues of the composite band must be identical at $\Gamma, K$, and $K^{\prime}$ [7]. But this cannot be satisfied, because $\mathrm{SB}_{1}$ contributes one $C_{3}$ eigenvalue $\left(=e^{i 2 \pi / 3}\right)$ at $\Gamma$, which can never have an equal counterpart at $K$ and $K^{\prime}$.

Conclusion.-The multicellular landscape, as enriched by crystalline symmetries, promises to be fertile ground for TIs that would naively be missed and deemed trivial. We have introduced two (not necessarily disjoint) classes of multicellular, Wannierizable TIs: rotation-invariant insulators with a returning Thouless pump, and Hopf insulators. For both classes, we have shown that bulk multicellularity (a) is a delicate topological invariant and (b) implies that the Hilbert space (on a half-infinite slab) cannot be Wannierized with WF centers identical to those of the bulk WFs. Whether (a) and (b) extend to all multicellular TIs is presently unanswered. Whether all delicate topological invariants are accompanied by bulk multicellularity is also unknown.

Our formulation of the RTP in terms of the Berry-Zak phase allows for a high-throughput search for material candidates. We have identified over 40 hexagonal magnetic space groups that allow a symmetry-protected RTP, which we tabulated in Sec. IX of the Supplemental Material [34]. After selecting materials in these space groups whose lowenergy bands satisfy the mutually disjoint symmetry condition, one would compute the Berry-Zak phase by standard first-principles techniques [61].

The multicellular Hopf insulator is already known to manifest higher-order topology, quantized surface magnetism [62], and quantized magnetoelectric polarizability [43]; it would be interesting to investigate if these properties extend to other multicellular and/or delicate topological insulators. Beyond band theory, we expect multicellularity to add a new chapter to the interplay between nonunicellular WFs, generalized Hubbard models, and exotic correlated phases [63-65].
We thank A. Bouhon for alerting us to Whitehead's formulation of the Hopf invariant and acknowledge a stimulating discussion with B.A. Bernevig about the obstructed atomic limit. Zhida Song helped to clarify a question on symmetry indicators. A. N. was supported by the Swiss National Science Foundation (SNSF) Grant No. 176877, and by Forschungskredit of the University of Zürich, Grant No. FK-20-098. T. N. acknowledges support from the European Research Council (ERC) under the European Union's Horizon 2020 Research and Innovation Programme (ERC-StG-Neupert-757867PARATOP) and from NCCR MARVEL funded by the SNSF. T. B. was supported by the SNSF Ambizione Grant No. 185806 . A. A. was supported by the Gordon and Betty Moore Foundation EPiQS Initiative through Grants No. GBMF 4305 and No. GBMF 8691 at the University of Illinois.

*anelson@physik.uzh.ch

†aalexan7@illinois.edu

[1] D. J. Thouless, Wannier functions for magnetic sub-bands, J. Phys. Condens. Matter 17, L325 (1984).

[2] T. Thonhauser and D. Vanderbilt, Insulator/Chern-insulator transition in the Haldane model, Phys. Rev. B 74, 235111 (2006).

[3] C. Brouder, G. Panati, M. Calandra, C. Mourougane, and N. Marzari, Exponential Localization of Wannier Functions in Insulators, Phys. Rev. Lett. 98, 046402 (2007).

[4] A. A. Soluyanov and D. Vanderbilt, Wannier representation of $\mathbb{Z}_{2}$ topological insulators, Phys. Rev. B 83, 035108 (2011).

[5] M. Taherinejad, K. F. Garrity, and D. Vanderbilt, Wannier center sheets in topological insulators, Phys. Rev. B 89, 115102 (2014).

[6] J. C. Budich, J. Eisert, E. J. Bergholtz, S. Diehl, and P. Zoller, Search for localized Wannier functions of topological band structures via compressed sensing, Phys. Rev. B 90, 115110 (2014).

[7] J. Höller and A. Alexandradinata, Topological Bloch oscillations, Phys. Rev. B 98, 024310 (2018).

[8] N. Read, Compactly supported Wannier functions and algebraic $k$-theory, Phys. Rev. B 95, 115309 (2017).

[9] A. Kitaev, Periodic table for topological insulators and superconductors, AIP Conf. Proc. 1134, 22 (2009).

[10] D. S. Freed and G. W. Moore, Twisted equivariant matter, Ann. Henri Poincare 14, 1927 (2013).

[11] J. Kruthoff, J. de Boer, J. van Wezel, C. L. Kane, and R.-J. Slager, Topological Classification of Crystalline Insulators through Band Structure Combinatorics, Phys. Rev. X 7, 041069 (2017).

[12] K. Shiozaki, M. Sato, and K. Gomi, Topological crystalline materials: General formulation, module structure, and wallpaper groups, Phys. Rev. B 95, 235425 (2017).

[13] H. C. Po, H. Watanabe, and A. Vishwanath, Fragile Topology and Wannier Obstructions, Phys. Rev. Lett. 121, 126402 (2018). 
[14] A. Bouhon, A. M. Black-Schaffer, and R.-J. Slager, Wilson loop approach to fragile topology of split elementary band representations and topological crystalline insulators with time-reversal symmetry, Phys. Rev. B 100, 195135 (2019).

[15] B. Bradlyn, Z. Wang, J. Cano, and B. A. Bernevig, Disconnected elementary band representations, fragile topology, and Wilson loops as topological indices: An example on the triangular lattice, Phys. Rev. B 99, 045140 (2019).

[16] D. V. Else, H. C. Po, and H. Watanabe, Fragile topological phases in interacting systems, Phys. Rev. B 99, 125122 (2019).

[17] Z.-D. Song, L. Elcoro, Y.-F. Xu, N. Regnault, and B. A. Bernevig, Fragile Phases as Affine Monoids: Classification and Material Examples, Phys. Rev. X 10, 031001 (2020).

[18] A. Bouhon, T. Bzdušek, and R.-J. Slager, Geometric approach to fragile topology beyond symmetry indicators, Phys. Rev. B 102, 115135 (2020).

[19] A. Alexandradinata, J. Höller, C. Wang, H. Cheng, and L. $\mathrm{Lu}$, Crystallographic splitting theorem for band representations and fragile topological photonic crystals, Phys. Rev. B 102, 115117 (2020).

[20] B. Bradlyn, L. Elcoro, J. Cano, M. G. Vergniory, Z. Wang, C. Felser, M. I. Aroyo, and B. A. Bernevig, Topological quantum chemistry, Nature (London) 547, 298 (2017).

[21] J. Cano, B. Bradlyn, Z. Wang, L. Elcoro, M. G. Vergniory, C. Felser, M. I. Aroyo, and B. A. Bernevig, Building blocks of topological quantum chemistry: Elementary band representations, Phys. Rev. B 97, 035139 (2018).

[22] H. C. Po, A. Vishwanath, and H. Watanabe, Symmetrybased indicators of band topology in the 230 space groups, Nat. Commun. 8, 50 (2017).

[23] A. Alexandradinata and J. Höller, No-go theorem for topological insulators and high-throughput identification of Chern insulators, Phys. Rev. B 98, 184305 (2018).

[24] J. Zak, Band representations and symmetry types of bands in solids, Phys. Rev. B 23, 2824 (1981).

[25] H. Bacry, Duals of crystallographic groups. Band and quasiband representations, Commun. Math. Phys. 153, 359 (1993).

[26] A. P. Schnyder, S. Ryu, A. Furusaki, and A. W. W. Ludwig, Classification of topological insulators and superconductors in three spatial dimensions, Phys. Rev. B 78, 195125 (2008).

[27] A. P. Schnyder, S. Ryu, A. Furusaki, and A. W. W. Ludwig, Classification of topological insulators and superconductors, AIP Conf. Proc. 1134, 10 (2009).

[28] N. Marzari and D. Vanderbilt, Maximally localized generalized Wannier functions for composite energy bands, Phys. Rev. B 56, 12847 (1997).

[29] A. Alexandradinata, X. Dai, and B. A. Bernevig, Wilsonloop characterization of inversion-symmetric topological insulators, Phys. Rev. B 89, 155114 (2014).

[30] G. H. Wannier, Wave functions and effective Hamiltonian for Bloch electrons in an electric field, Phys. Rev. 117, 432 (1960).

[31] J. Zak, Berry's Phase for Energy Bands in Solids, Phys. Rev. Lett. 62, 2747 (1989).

[32] R. D. King-Smith and D. Vanderbilt, Theory of polarization of crystalline solids, Phys. Rev. B 47, 1651 (1993).

[33] D. J. Thouless, Quantization of particle transport, Phys. Rev. B 27, 6083 (1983).
[34] See Supplemental Material at http://link.aps.org/supplemental/ 10.1103/PhysRevLett.126.216404 for (a) comments on obstructed atomic limit, (b) information about the three- and fourband models, (c) proof of the RTP-Hopf relation, (d) proof of the RTP multicellularity, (e) analysis of the surface band representations and obstruction principle, and (f) classification of RTP-compatible hexagonal magnetic space groups, which includes Refs. [35-41].

[35] W. P. Su, J. R. Schrieffer, and A. J. Heeger, Solitons in Polyacetylene, Phys. Rev. Lett. 42, 1698 (1979).

[36] J. Ahn, D. Kim, Y. Kim, and B.-J. Yang, Band Topology and Linking Structure of Nodal Line Semimetals with $Z_{2}$ Monopole Charges, Phys. Rev. Lett. 121, 106403 (2018).

[37] A. Nelson, T. Bzdušek, T. Neupert, and A. Alexandradinata, Delicate topology protected by rotation symmetry: Crystalline Hopf insulators and beyond (to be published).

[38] X. L. Qi, Y. S. Wu, and S. C. Zhang, Topological quantization of the spin Hall effect in two-dimensional paramagnetic semiconductors, Phys. Rev. B 74, 085308 (2006).

[39] X.-Q. Sun, S.-C. Zhang, and T. Bzdušek, Conversion Rules for Weyl Points and Nodal Lines in Topological Media, Phys. Rev. Lett. 121, 106402 (2018).

[40] L. Elcoro, B. J. Wieder, Z. Song, Y. Xu, B. Bradlyn, and B. A. Bernevig, Magnetic topological quantum chemistry, arXiv:2010.00598.

[41] Y. Xu, L. Elcoro, Z.-D. S. Song, B. J. Wieder, M. G. Vergniory, N. Regnault, Y. Chen, C. Felser, and B. A. Bernevig, High-throughput calculations of magnetic topological materials, Nature (London) 586, 702 (2020).

[42] J. D. Cloizeaux, Analytical properties of $n$-dimensional energy bands and Wannier functions, Phys. Rev. 135, A698 (1964).

[43] A. Alexandradinata, A. Nelson, and A. A. Soluyanov, Teleportation of Berry curvature on the surface of a Hopf insulator, Phys. Rev. B 103, 045107 (2021).

[44] H. Hopf, Über die Abbildungen der dreidimensionalen Sphäre auf die Kugelfläche, Math. Ann. 104, 637 (1931).

[45] L. Pontrjagin, A classification of mappings of the threedimensional complex into the two-dimensional sphere, Mat. Sb. 9, 331 (1941), http://m.mathnet.ru/sm6073.

[46] R. Kennedy, Topological Hopf-Chern insulators and the Hopf superconductor, Phys. Rev. B 94, 035137 (2016).

[47] F. N. Ünal, A. Eckardt, and R.-J. Slager, Hopf characterization of two-dimensional Floquet topological insulators, Phys. Rev. Research 1, 022003(R) (2019).

[48] J. E. Moore, Y. Ran, and X.-G. Wen, Topological Surface States in Three-dimensional Magnetic Insulators, Phys. Rev. Lett. 101, 186805 (2008).

[49] F. Wilczek and A. Zee, Linking Numbers, Spin, and Statistics of Solitons, Phys. Rev. Lett. 51, 2250 (1983).

[50] M. V. Berry, Quantal phase factors accompanying adiabatic changes, Proc. R. Soc. A 392, 45 (1984).

[51] J. H. C. Whitehead, An expression of Hopf's invariant as an integral, Proc. Natl. Acad. Sci. U.S.A. 33, 117 (1947).

[52] E. Khalaf, W. A. Benalcazar, T. L. Hughes, and R. Queiroz, Boundary-obstructed topological phases, Phys. Rev. Research 3, 013239 (2021).

[53] A. M. Essin, J. E. Moore, and D. Vanderbilt, Magnetoelectric Polarizability and Axion Electrodynamics in Crystalline Insulators, Phys. Rev. Lett. 102, 146805 (2009). 
[54] X.-L. Qi, T. L. Hughes, and S.-C. Zhang, Topological field theory of time-reversal invariant insulators, Phys. Rev. B 78, 195424 (2008).

[55] A. M. Essin, J. E. Moore, and D. Vanderbilt, Magnetoelectric Polarizability and Axion Electrodynamics in Crystalline Insulators, Phys. Rev. Lett. 102, 146805 (2009); Erratum, Phys. Rev. Lett. 103, 259902 (2009).

[56] A. M. Essin, A. M. Turner, J. E. Moore, and D. Vanderbilt, Orbital magnetoelectric coupling in band insulators, Phys. Rev. B 81, 205104 (2010).

[57] A. Malashevich, I. Souza, S. Coh, and D. Vanderbilt, Theory of orbital magnetoelectric response, New J. Phys. 12, 053032 (2010).

[58] D. Vanderbilt, Berry Phases in Electronic Structure Theory: Electric Polarization, Orbital Magnetization and Topological Insulators (Cambridge University Press, Cambridge, England, 2018).

[59] C. Fang, M. J. Gilbert, and B. A. Bernevig, Bulk topological invariants in noninteracting point group symmetric insulators, Phys. Rev. B 86, 115112 (2012).

[60] L. Elcoro, B. Bradlyn, Z. Wang, M. G. Vergniory, J. Cano, C. Felser, B. A. Bernevig, D. Orobengoa, G. de la Flor, and
M. I. Aroyo, Double crystallographic groups and their representations on the Bilbao crystallographic server, J. Appl. Crystallogr. 50, 1457 (2017).

[61] D. Gresch, G. Autès, O. V. Yazyev, M. Troyer, D. Vanderbilt, B. A. Bernevig, and A. A. Soluyanov, Z2pack: Numerical implementation of hybrid Wannier centers for identifying topological materials, Phys. Rev. B 95, 075146 (2017).

[62] P. Zhu, T. L. Hughes, and A. Alexandradinata, Quantized surface magnetism and higher-order topology: Application to the Hopf insulator, Phys. Rev. B 103, 014417 (2021).

[63] J. S. Hofmann, E. Berg, and D. Chowdhury, Superconductivity, pseudogap, and phase separation in topological flat bands, Phys. Rev. B 102, 201112(R) (2020).

[64] J. Kang and O. Vafek, Strong Coupling Phases of Partially Filled Twisted Bilayer Graphene Narrow Bands, Phys. Rev. Lett. 122, 246401 (2019).

[65] V. Peri, Z.-D. Song, B. A. Bernevig, and S. D. Huber, Fragile Topology and Flat-band Superconductivity in the Strong-Coupling Regime, Phys. Rev. Lett. 126, 027002 (2021). 